attractor. Our experiments confirm the physical relevance of this attractor, in spite of viscous and nonlinear effects. A standing wave is initially excited by the parametric instability, turning into a propagating wave in the permanent regime. A usual boundary forcing should only produce the latter regime, with permanent excitation at large scale, and energy transfers towards small scales along the attractor ${ }^{9}$. The system also has a broad-band response in frequency, set by the interval of existence of the attractor. This behaviour contrasts dramatically with the narrow peaks of usual resonances, whose width is set by dissipative effects.

Similar internal wave attractors could be predicted, by ray tracing, in ocean basins or lakes. Waves must be amplified by focusing at their contact with the sloping bottom. This should determine zones of intense mixing, favouring life, by providing a pathway for the transport of nutrients and rare metals from the bottom to the photic zone $\mathrm{e}^{10,11}$. This focusing process has often been invoked $^{12-14}$, but without considering its localization on a global attractor. Such localization depends of course on the wave frequency. In the ocean, important contributions at the tidal frequency should be expected, but, unlike our experiments, wind-related frequencies are simultaneously excited, and the widest frequency 'windows', corresponding to an attractor related to the particular geometry, may show up. The dressing of the attractor by the wave solution is clearly understood in our two-dimensional case, but a (non-trivial) three-dimensional generalization must be sought for natural basins.

Our results could also be relevant to an experiment ${ }^{15}$ that showed inertial oscillations in a fluid contained in a truncated cone rotating around its axis (where axisymmetric modes are excited by a sinusoidal modulation of the rotation rate). The spectral response was in this case interpreted in terms of resonance peaks of standing waves (presumably broadened by viscous effects). We note, however, that the spectral 'peaks' and 'valleys' coincide remarkably well with the regions of strong and weak convergence rates (obtained as an appropriate cross-section in Fig. 2 for $d=-0.478$ ): so we are led to conclude that the broadness of these response 'peaks' may well reflect a genuine, finite bandwidth of the response rather than a viscously spread-out resonance spike.

The problem of confined inviscid inertial oscillations, as occur in a spherical shell, is also ill-posed ${ }^{16}$ (unlike in a sphere). Smooth eigenmodes can be computed by introducing a small viscosity ${ }^{17}$, but better physical insight is expected in terms of wave attractors. The simplest attractors would again show up as wide resonance bands, whose width is independent of dissipative effects. This may be relevant to analysing inertial oscillations of the Earth core ${ }^{18,19}$ and to the stability of liquid-filled, rotating spacecraft ${ }^{20}$.

Received 19 December 1996; accepted 17 June 1997

1. Maas, L. R. M. \& Lam, F.-P. A. Geometric focusing of internal waves. J. Fluid Mech. 300, 1-41 (1995). 2. Görtler, H. Über eine Schwingungserscheinung in Flüssigkeiten mit stabiler Dichteschichtung. $Z$. Angew. Math. Mech. 23, 65-71 (1943).

3. Berry, M. V. Regularity and chaos in classical mechanics, illustrated by three deformations of a circular 'billiard'. Eur. J. Phys. 2, 91-102 (1981).

4. Wedderburn, E. M. The temperature seiche. I-IV. Phil. Trans. R. Soc. Edinb. 47, 619-642 (1911).

5. Courant, R. \& Hilbert, D. Methoden der Mathematischen Physik Vol. II (Springer, Berlin, 1937).

6. McEwan, A. D. \& Robinson, R. M. Parametric instability of internal gravity waves. J. Fluid Mech. 67, 667-687 (1975)

7. Benielli, D. \& Sommeria, J. Excitation of internal waves and stratified turbulence by parametric instability. Dyn. Atmos. Oceans 23, 335-343 (1996).

8. Joseph, B. Chaotic mixing by internal inertia-gravity waves. Phys. Fluids 9, 945-962 (1997).

9. Phillips, O. M. Energy transfer in rotating fluids by reflection of inertial waves. Phys. Fluids 6, 513-520 (1963).

10. Steele, J. H. Spatial Pattern in Plankton Communities (Plenum, New York, 1978).

11. Holligan, P. M., Pingree, R. D. \& Mardell, G. T. Oceanic solitons, nutrient pulses and phytoplankton growth. Nature 314, 348-350 (1985).

12. Eriksen, C. Implications of ocean bottom reflections for internal wave spectra and mixing. J. Phys. Oceanogr. 15, 1145-1156 (1985).

13. Garrett, C. J. R. \& Gilbert, D. in Small-scale Turbulence and Mixing in the Ocean (eds Nihoul, J. C. J. \& Jamart, B. M.) 405-423 (Elsevier, Amsterdam, 1988).

14. Ivey, G. N. \& Nokes, R. I. Vertical mixing due to the breaking of critical internal waves on sloping boundaries. J. Fluid. Mech. 204, 479-500 (1989).

15. Beardsley, R. C. An experimental study of inertial waves in a closed cone. Stud. Appl. Math. 49, 187 196 (1970).

16. Stewartson, K. \& Rickard, J. A. Pathological oscillations of a rotating fluid. J. Fluid Mech. 35, 759-773 (1969).
17. Rieutord, M. Inertial modes in the liquid core of the earth. Phys. Earth Planet. Inter. 91, 41-46 (1995). 18. Melchior, P. \& Ducarme, B. Detection of inertial gravity oscillations in the Earth's core with a superconducting gravimeter at Brussels. Phys. Earth Planet. Inter. 42, 129-134 (1986).

19. Aldridge, K. D. \& Lumb, L. I. Inertial waves identified in the Earth's fluid outer core. Nature 325, 421423 (1987).

20. Kobine, J. J. Inertial wave dynamics in a rotating and precessing cylinder. J. Fluid Mech. 303, 233-252 (1995).

21. Magaard, L. Ein Beitrag zur Theorie der internen Wellen als Störungen geostrophischer Strömungen. Deutsche Hydrogr. Z. 21, 241-278 (1968).

22. Cacchione, D. \& Wunsch, C. Experimental study of internal waves over a slope. J. Fluid Mech. 66, 223 239 (1974).

Acknowledgements. We thank G. van der Plas and S. Dalziel for help with post-processing of the data, and C. Staquet and B. Voisin for suggestions.

Correspondence should be addressed to L.R.M.M. (e-mail: maas@nioz.nl).

\section{Discovery of a reactive azeotrope}

\section{W. Song, R. S. Huss, M. F. Doherty \& M. F. Malone}

Department of Chemical Engineering, Goessmann Laboratory,

University of Massachusetts, Amherst, Massachusetts 01003-3110, USA

Mixtures are azeotropic if they can be distilled (or condensed) without a change of composition ${ }^{1}$. The existence of azeotropes in multicomponent mixtures in the absence of chemical reactions is well understood phenomenologically ${ }^{2,3}$ and theoretically $y^{4,5}$. Azeotropes place a fundamental limit on the compositions attainable in mixtures by fractional distillation, but they can in some cases be 'broken' by carrying out chemical reaction and separation simultaneously rather than sequentially ${ }^{6-9}$. Here we report the discovery of a boiling state of constant composition and temperature in a mixture of acetic acid, isopropanol, isopropyl acetate and water that is simultaneously in both reaction and phase equilibrium. These states, which we call reactive azeotropes, were predicted recently ${ }^{10,11}$. Without reaction, the mixture exhibits three twocomponent azeotropes, one three-component azeotrope but no four-component azeotrope; the last appears only under equilibrium reaction conditions. These findings may constrain technologies in which reaction and separation are conducted simultaneously, for example by limiting the conditions under which an azeotrope can be broken by chemical reactions to yield a high-purity product. In other cases the presence of a reactive azeotrope may be advantageous'.

Without chemical reaction, a mixture of acetic acid, isopropanol, isopropyl acetate and water has three binary azeotropes and a ternary azeotrope as listed in Table 1. There is no four-component azeotrope in this mixture. An acid-catalysed esterification reaction occurs in this mixture:

$$
\mathrm{CH}_{3} \mathrm{COOH}+\text { iso- } \mathrm{C}_{3} \mathrm{H}_{7} \mathrm{OH} \leftrightarrow \mathrm{CH}_{3} \mathrm{COOC}_{3} \mathrm{H}_{7}+\mathrm{H}_{2} \mathrm{O}
$$

This reaction occurs slowly; the rate can be increased substantially by the addition of a strong acid catalyst. The non-reactive binary azeotropes between water and isopropanol and between isopropyl acetate and isopropanol will also exist in the reacting mixture. However, a binary mixture of isopropyl acetate and water, as well as any mixture containing three of the components, will react and the corresponding azeotropes are absent.

A recent and general theory for mixtures in simultaneous vapour-liquid and chemical reaction equilibrium revealed the possibility of a state with a constant boiling temperature and constant, though not equal, vapour and liquid compositions ${ }^{10,11}$. In a reacting, boiling liquid each component has a rate of vaporization and a rate of creation or loss due to reaction. When the net rate for each component is zero, an azeotrope occurs. However, the existence of a reactive azeotrope has not been confirmed experimentally.

The theory ${ }^{10,11}$ provides a general transformation of variables 
a Isopropanol $\left(82.25^{\circ} \mathrm{C}\right) \quad\left(80.06^{\circ} \mathrm{C}\right)$

Isopropyl acetate $\left(88.5^{\circ} \mathrm{C}\right)$

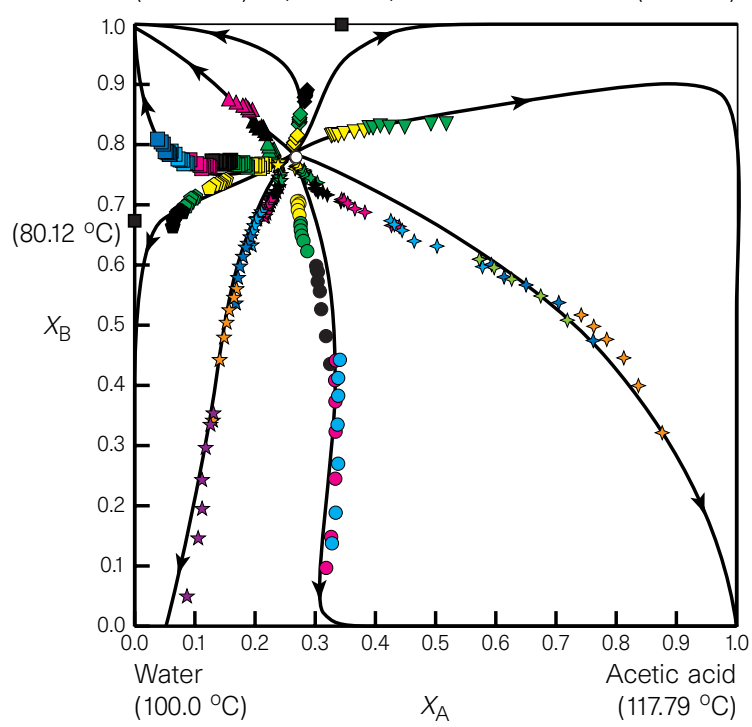

Figure $1 \mathrm{a}$, Comparison of experiments and calculated residue curves; $X_{\mathrm{A}}$ and $X_{\mathrm{B}}$ are composition variables (see text). The calculated residue curves are shown by the solid lines. The arrows show the direction of increasing time and temperature. The shapes of the plotting symbols show experiments within a series; the colours of the symbols distinguish different runs within that series. The run closest to the azeotrope is shown with open circles. The temperatures shown are those predicted by the model. The calculated curves were generated with the NonRandom Two Liquid (NRTL) mode ${ }^{14}$ for activities in the liquid phase, including association of acetic acid in the vapour phase. Parameters for the model were taken from the literature ${ }^{12}$ except for the isopropanol-isopropyl acetate and water-isopropyl acetate interactions, which we estimated from the UNIFAC group contribution method ${ }^{15}$ optimized for the locations of the non-reactive

from mole fractions $(x)$ to a new set of composition variables $(X)$. This takes into account the reduction in degrees of freedom due to chemical reaction and provides a natural coordinate system. In the mixture studied here, the transformed variables represent the compositions in terms of the total amount of acetic acid and isopropanol, including the equivalent amounts present in the other two components. These are:

$$
\begin{aligned}
& X_{\mathrm{A}}=x_{\text {acetic acid }}+x_{\mathrm{i}-\text { propyl acetate }} \\
& X_{\mathrm{B}}=x_{\mathrm{i}-\text { propanol }}+x_{\mathrm{i}-\text { propyl acetate }} \\
& X_{\mathrm{C}}=x_{\text {water }}-x_{\mathrm{i}-\text { propyl acetate }}
\end{aligned}
$$

The feasible composition space is the square shown in Fig. 1a. Each vertex of the square is a pure component, each edge is a non-reactive binary mixture, and any point inside the square is a four-component reactive mixture.

Figure 1a also shows residue curves, which represent the liquid composition paths in an isobaric open evaporation. For a single chemical reaction, these are the solutions of

$$
\frac{\mathrm{d} X_{i}}{\mathrm{~d} \xi}=Y_{i}-X_{i}
$$

for $i=1,2, \ldots, c-r-1$ where $c$ is the number of chemical species, $r$ is the number of independent chemical reactions, and $\xi$ is a dimensionless time. The transformed liquid and vapour compositions, $X$ and $Y$, are related by phase and chemical-reaction equilibrium.

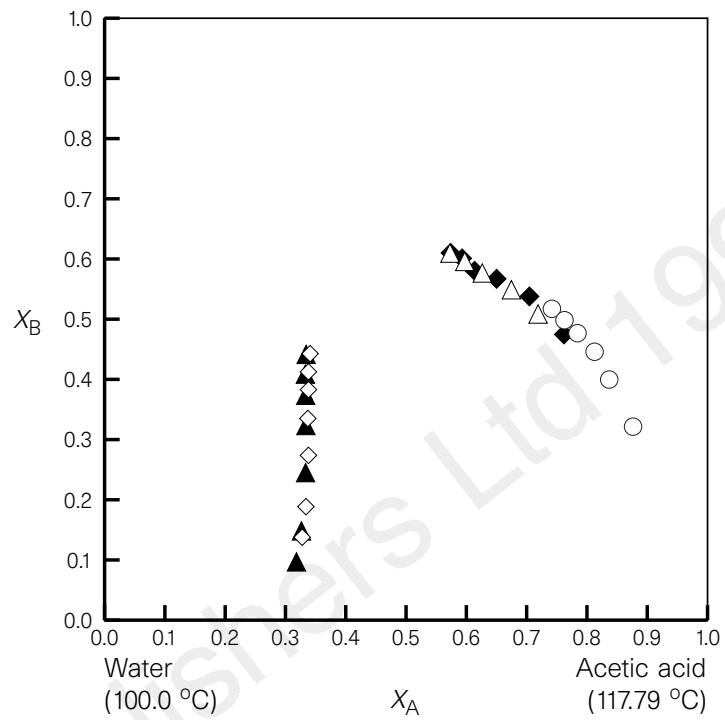

azeotropes listed in Table 1. We used a constant value of 8.7 for the equilibrium constant for the reaction (see equation (1)) as reported by Lee and Kuo ${ }^{13}$. b. Replicated experiments. The filled diamonds and triangles show the two original experiments and the open diamonds and triangles show the replications. The open circles are a run beginning from a composition near the last measured value of the original series. In the original and replicated experiments, measurements were taken at the same time intervals. We note that the replicated runs might be slightly shifted in time owing to small differences in vapour removal rate. However, as demonstrated in the figure, the trajectories of compositions correspond quite closely to those expected for systems with dynamics that follow autonomous models such as equation (3).

Residue curves begin and end at azeotropes or pure components and are directed from low to high temperatures. In Fig. 1a, all of the calculated residue curves (solid lines) originate from a point inside the figure where

$$
X_{i}=Y_{i}
$$

for $i=1,2, \ldots, c-r-1$. This point is a minimum-boiling, reactive azeotrope with a molar liquid composition of $5.30 \%$ acetic acid, $56.55 \%$ isopropanol, $21.87 \%$ isopropyl acetate and $16.28 \%$ water at a temperature of $78.99^{\circ} \mathrm{C}$ and a vapour composition of $0.33 \%$ acetic acid, $51.58 \%$ isopropanol, $26.84 \%$ isopropyl acetate and $21.25 \%$ water. The corresponding transformed compositions are $X_{\mathrm{A}}=Y_{\mathrm{A}}=0.2717$ and $X_{\mathrm{B}}=Y_{\mathrm{B}}=0.7842$.

To test this prediction, we measured the composition changes in a liquid undergoing simultaneous open evaporation and chemical reaction. A solid acidic resin (Amberlyst 15, Rohm \& Haas Co., Philadelphia, PA) was used as a catalyst. The liquid compositions were measured periodically by removing small samples for analysis by gas chromatography (Hewlett-Packard 5890 Series II gas chromatograph, TC detector, HP-INNoWax column). For gas chromatography measurements, $0.1 \mathrm{ml}$ of liquid sample was diluted with $20 \mathrm{ml}$ of anhydrous diethylether. A small quantity $(1 \mu \mathrm{l})$ of the diluted solution was injected for analysis. The response of the gas chromatograph was calibrated for each of the four components of the mixture. At the beginning of an experiment, the mixture was refluxed with catalyst until the temperatures of both the liquid and vapour phases were stabilized. Vapour was then removed continuously and the liquid compositions were measured as a function of 


\section{Table 1 Azeotropes at 1 atm}

Mole fractions

\begin{tabular}{|c|c|c|c|c|}
\hline $\begin{array}{l}\text { Boiling } \\
\text { temperature }\left({ }^{\circ} \mathrm{C}\right)\end{array}$ & $\begin{array}{l}\text { Acetic } \\
\text { acid }\end{array}$ & Isopropanol & $\begin{array}{l}\text { Isopropyl } \\
\text { acetate }\end{array}$ & Water \\
\hline 75.5 & 0.00 & 0.1377 & 0.4737 & 0.3887 \\
\hline 76.6 & 0.00 & 0.00 & 0.5980 & 0.4020 \\
\hline 80.1 & 0.00 & 0.6480 & 0.3520 & 0.00 \\
\hline 80.1 & 0.00 & 0.6873 & 0.00 & 0.3127 \\
\hline
\end{tabular}

Data reported by Horsley ${ }^{3}$

time. Eventually, the amount of liquid residue was small enough to preclude sampling, ending the experiment. Subsequent experiments, beginning at compositions close to the end point of the previous experiment, were necessary in most cases to get a clear measure of each residue curve.

We studied the effect of catalyst concentration by using the same initial composition of the mixture with different amounts of catalyst. Our interest here is in the limiting case approaching phase and reaction equilibrium so we sought to find a large amount of catalyst, a low rate of vapour withdrawal, and the appropriate start-up procedure to approach this condition. With $27.5 \mathrm{~g}$ of catalyst $\left(0.135 \mathrm{~mol} \mathrm{H}{ }^{+}\right)$in an initial volume of $306 \mathrm{ml}$ liquid, the theoretical residue curve is approached closely. The similarity between the experimental residue curves for 22.0 and $27.5 \mathrm{~g}$ of catalyst showed an approach to this limiting case. Based on these results, we used $30.0 \mathrm{~g}$ catalyst $\left(0.147 \mathrm{~mol} \mathrm{H}^{+}\right)$in an initial volume of $306 \mathrm{ml}$ for all experiments reported here. Using a similar approach, we determined a heating rate low enough to ensure that the results are independent of the rate of mass transfer between liquid and vapour phases. In our experiment, this was achieved in a time of $\sim 6$ hours to remove $\sim 75 \%$ of the initial liquid or an average vapour removal rate of $\sim 38 \mathrm{ml} \mathrm{h}^{-1}$.

A series of nine experiments with initial conditions in the neighbourhood of the calculated azeotrope were performed in a total of 39 runs; each set in a series was intended to represent one residue curve; successive runs were necessary in most cases to show a significant portion of each curve. The results are shown in Fig. 1a. The open circles indicate the position of the reactive azeotrope as closely as it could be determined; these points are nearly overlapping as predicted. This single run consists of five data points taken over a span $\sim 4$ hours, during which time the compositions remained nearly constant and the boiling temperature varied by $<0.1^{\circ} \mathrm{C}$. The measured molar liquid composition of the reactive azeotrope was $5.4 \%$ acetic acid, $56.5 \%$ isopropanol, $21.4 \%$ isopropyl acetate and $16.7 \%$ water at a temperature of $78.6^{\circ} \mathrm{C}$, with a corresponding vapour composition of $0.0 \%$ acetic acid, $49.1 \%$ isopropanol, $27.0 \%$ isopropyl acetate and $23.9 \%$ water. The corresponding transformed compositions are $X_{\mathrm{A}}=0.268, X_{\mathrm{B}}=0.779$ and $Y_{\mathrm{A}}=0.270$, $Y_{\mathrm{B}}=0.761$.

We assessed the reproducibility of the results by repeating two runs and found the data to be highly reproducible, as shown in Fig. 1b.

These results demonstrate the existence of a reactive azeotrope not present in the non-reactive mixture. This confirms the predictions of a model based on classical thermodynamics and provides a basis for predicting the behaviour of other mixtures. For example, we also predict reactive azeotropes in the esterification of acetic acid with $n$-propanol or iso-butanol, as well as a near reactive azeotrope in the reaction of methanol and isobutene to form methyl $t$-butyl ether (MTBE). In the production of MTBE the 'reactive azeotrope' is predicted to contain a substantial amount of MTBE and is responsible for making reactive distillation the preferred process technology ${ }^{9}$. We believe that reactive azeotropes are common and for some systems can determine the optimal choice of process technology. As a further example, the manufacture of methyl acetate uses reactive distillation, because reaction eliminates the azeotrope between water and methyl acetate that otherwise limits the purity of the product ${ }^{8,9}$. But our results show that a similar process will not work for isopropyl acetate because of the existence of a reactive azeotrope in the four-component reactant/product mixture.

Received 20 January; accepted 24 June 1997.

1. Rowlinson, J. S. Liquids and Liquid Mixtures 2nd edn (Butterworth, London, 1969).

2. Lecat, M. Tables Azeotropiques: I. Azeotropes Binares Orhthobares (l'Auteur, Bruxelles, 1949).

3. Horsley, L. H. Azeotropic Data-III (Am. Chem. Soc., Washington DC, 1973).

4. Malesinski, W. Azeotropy and Other Theoretical Problems of Vapour-Liquid Equilibrium (Interscience, New York, 1965).

5. Swietoslawski, W. Azeotropy and Polyazeotropy (Pergamon, Warsaw, 1963).

6. Xu, X., Zhu, B., Chen, H. \& Bai, G. Reactive distillation (I). Shiyou Huagong (Petrochemical Technology) 14, 480-486 (1985)

7. Xu, X., Zhu, B., Chen, H. \& Bai, G. Reactive distillation (II). Shiyou Huangong (Petrochemical Technology) 14, 550-555 (1985).

8. Agreda, V. H. \& Partin, L. R. Reactive distillation process for the production of methyl acetate. US Patent No. 4435595 (to Eastman Kodak Company) (1984).

9. Doherty, M. F. \& Buzad, G. Reactive distillation by design. Trans. Inst. Chem. Eng. A 70, 448-458 (1992).

10. Barbosa, D. \& Doherty, M. F. Theory of phase diagrams and azeotropic conditions for two-phase reactive systems. Proc. R. Soc. Lond. A 413, 443-458 (1987).

11. Ung, S. \& Doherty, M. F. Necessary and sufficient conditions for reactive azeotropes in multireaction mixtures. Am. Inst. Chem. Eng. J. 41, 2383-2392 (1995).

12. Gmehling, J. \& Onken, U. Vapor-Liquid Equilibrium Data Collection (DECHEMA Chemistry Data Ser., Frankfurt, 1977).

13. Lee, L-S. \& Kuo, M-Z. Phase and reaction equilibrium of the acetic acid-isopropanol-isopropyl acetate-water system at $760 \mathrm{~mm} \mathrm{Hg}$. Fluid Phase Equil. 123, 147-165 (1996).

14. Renon, H. \& Prausnitz, J. M. Local compositions in thermodynamic excess functions for liquid mixtures. Am. Inst. Chem. Eng. J. 14, 135-144 (1968).

15. Fredenslund, A., Gemehling, J. \& Rasmussen, P. Vapor-Liquid Equilibria Using UNIFAC (Elsevier, Amsterdam, 1977).

Acknowledgements. This work was supported by National Science Foundation and the National Environmental Technology Institute.

Correspondence should be addressed to M.F.D. (e-mail: mdoherty@ecs.umass.edu).

\section{Decadal predictability of North Atlantic sea surface temperature and climate}

\section{R. T. Sutton ${ }^{\star} \dagger \&$ M. R. Allen ${ }^{\star} \ddagger$}

* Atmospheric Oceanic and Planetary Physics, Clarendon Laboratory, University of Oxford, Parks Road, Oxford OX1 3PU, UK

$\dagger$ Centre for Global Atmospheric Modelling, Department of Meteorology, University of Reading, PO Box 243, Earley Gate, Reading RG6 6BB, UK $\ddagger$ Space Science Department, Rutherford Appleton Laboratory,

Chilton OX11 0QX, UK

The weather at middle latitudes is largely unpredictable more than a week or so in advance, whereas fluctuations in the ocean may be predictable over much longer timescales. If decadal fluctuations in North Atlantic sea surface temperature ${ }^{1-6}$ could $^{-}$ be predicted, it might be possible to exploit their influence on the atmosphere $^{7-10}$ to forecast decadal fluctuations in climate ${ }^{11}$. Here we report analyses of shipboard observations that indicate significant decadal predictability of North Atlantic sea surface temperature, arising from the advective propagation of sea-surfacetemperature anomalies ${ }^{4}$ and the existence of a regular period of 12-14 years in the propagating signals. The same timescale can be identified in a dipole-like pattern of North Atlantic sea-level pressure variability ${ }^{1,7,12}$. We propose a mechanism which may connect these oceanic and atmospheric fluctuations, possibly as part of a coupled ocean-atmosphere mode of variability ${ }^{7}$. Our results are encouraging for the prospects of forecasting natural fluctuations in the climate of the North Atlantic region several years in advance.

Several authors have suggested that much of the decadal variability in North Atlantic sea surface temperature (SST) can be explained as a local oceanic response to atmospheric variability ${ }^{1,3,13}$, 